\section{Psychogeriatric Liaison Referrals}

SIR: We read with great interest the paper by Poynton (Journal, January 1988, 152, 45-47) regarding psychogeriatric liaison referrals at Guy's. Although we were not entirely sure of Dr Poynton's expectations, she was clearly disappointed with the impact of their new service even though there was a significant increase in referrals in their first year. She states that the referral rate for the elderly before and after the service started remained below the $1.3 \%$ found with younger patients, yet (unless we are mistaken) she shows an increase in the elderly referral rate from $0.64 \%$ before to $1.4 \%$ after the service was initiated.

We are currently analysing our own experience at the Royal Liverpool Hospital, where there has been a psychogeriatric liaison service for 7 years. Our clinical situation resembles Guy's in serving a teaching hospital with only acute beds, having a deprived inner-city catchment area, and all our consultations being provided by the consultant or senior registrar in psychogeriatrics. Dr Poynton points out that had her study periods been longer there may have been more obvious differences reflecting a "gradual change in practice". We therefore thought it would be of interest to report our own first and most recent years of experience and contrast these with that reported from Guy's.

Considering 12-monthly periods, our first year (1981-82) produced 54 referrals $(0.7 \%$ over-65 nonpsychiatric discharges) and our most recent period (1987-88) 166 referrals (1.96\% over-65 discharges) representing a highly significant increase $\left(\chi^{2}=42.7\right.$, $P<0.001$, raw data). What is more interesting are the changes in the pattern of diagnostic groups referred. Using the diagnoses employed by Dr Poynton and comparing our first year with that of Guy's, we saw more cases of 'dementia' $(P<0.001)$ and fewer 'other psychiatric disorders' $(P<0.01)$ but statistically similar proportions of 'depression', 'acute confusion' and 'no psychiatric disorder'.

Comparing our own figures for 1981-82 with 1987-88, we find that a substantial change in diagnoses has occurred, such that we now see significantly more cases of 'depression' $(P<0.01)$ but fewer of 'acute confusion' $(P<0.01)$ and 'no psychiatric disorder' $(P<0.02)$. The proportion of 'dementias' and 'other psychiatric disorders' remain unchanged, although the absolute number of dementias has increased threefold while the number of depression cases has increased ninefold.

These changes only became apparent after approximately 3 years, and Dr Poynton's pessimism may, therefore, be premature. Although we realise the limitations of the data as presented, it does represent a gradual and consistent pattern that has emerged over this time. It would be interesting to consider the reasons for this, but any meaningful discussion is prohibited by shortage of space. We would, however, suggest that one major factor responsible for this change is the educational effect of a specialist liaison service in altering the perception and awareness of psychiatric disorders in old age by non-psychiatrists. Thus we may explain a greatly increased number of depressions detected and referred, but no concomitant increase of acute confusions and no psychiatric disorder which should, generally, be managed by the referring agency themselves. We have particularly tried to emphasise to other departments and undergraduates the prevalence of depression on acute wards and the recognition of acute confusion with its need of medical investigation and treatment. This effect takes time to emerge and requires commitment from an enthusiastic department persistently and vigorously getting the message across in both clinical and non-clinical settings. We believe the effort is being made in Liverpool to establish this situation, and have no doubt that similar enthusiasm obtains at Guy's.

We support Dr Poynton's call for further research, particularly in establishing that the right patients are being referred while the non-referred are also recognised and managed appropriately.

Education must be an essential and priority component of a liaison service if we are to improve the quality of practice in the care of the elderly. It is also imperative that we teach the acute sector to recognise and manage, not simply refer, the majority of the 30 $70 \%$ of psychiatric morbidity on their wards, for even superhuman enthusiasm and commitment would not enable us to cope with $2500-6000$ referrals per year.

\section{N. ANDERSON \\ R. M. PHILPOTT}

Royal Liverpool Hospital

K. C. M. WILSON

\section{Prescot Street}

Liverpool L7 8XP

\section{Failure to Convulse with ECT}

SIR: The article by Sharpe \& Andrew (Journal, January 1988, 152, 134-136) draws attention to an important problem associated with ECT administration. In response, Pippard \& Russell (Journal, May 1988, 152, 712-713) have noted the critical role of adequate electrical stimulation in administering ECT. Recent work in our department sheds further light on two other issues touched upon by Drs Sharpe \& Andrew. 
The authors mention the use of caffeine as a possible measure for lowering seizure threshold. Subsequent to the case report which they cite (Shapira et al, 1985), we have evaluated the effect of pre-ECT administration of caffeine sodium benzoate on seizure parameters in a controlled study (Shapira et al, 1987). The results supported our original observation and showed a significant increase in seizure duration during treatments preceded by caffeine administration. Caffeine did not induce untoward haemodynamic effects, nor a greater degree of cognitive impairment than is normally associated with ECT, and was subjectively well-tolerated. These findings have been borne out by other studies (e.g. Hinkle $e t$ al, 1987). The patient reported by Drs Sharpe \& Andrew eventually remitted following lithium supplementation of ongoing treatment with amitryptiline and chlorpromazine. We have recently reported (Shapira et al, 1988) seven patients who were unresponsive to ECT and eventually remitted following lithium supplementation of tricyclic antidepressant (TCA) treatment. Two had not responded to this combination prior to ECT. Four of these patients were administered ECT in our hospital; they all had seizures exceeding $25 \mathrm{~s}$ in length during their courses of 11 or more bilateral treatments. Three reports by other authors (e.g. Nelson \& Mazure, 1986) mention cases in which lithium supplementation of TCA induced remission in patients unresponsive to ECT. We therefore suggest that lithium supplementation be considered in TCA non-responders prior to referral for ECT.

This work was supported in part by NIMH grant No. 40734.

Jerusalem Mental Health Center-Ezrath Nashim

BARUCH SHAPIRA SETH KindLeR BERNARD LERER PO Box 140

Jerusalem

Israel

\section{References}

Hinkle, P. E., Coffey, C. E., Weiner, R. D., Cress, M. \& Christison, C. (1987) Use of caffeine to lengthen seizures in ECT American Journal of Psychiatry, 144, 1143-1148.

NeLSON, J. \& MAZURE, C. M. (1986) Lithium augmentation in psychotic depression refractory to combined drug treatment. American Journal of Psychiatry, 143, 363-366.

Shaptra, B., Zohar, J., Newman, M., Drexler, H. \& Belmaker, R. (1985) Potentiation of seizure, length and clinical response to electroconvulsive therapy by caffeine pretreatment: a case report. Convulsive Therapy, 1, 50-60.

-, Lerer, B., Gilboa, D., Drexler, H., Kugelmass, S. \& Calev, A. (1987) Facilitation of ECT by caffeine pretreatment. American Journal of Psychiatry, 144, 1199-1202.

-, Kindler, S. \& Lerer, B. (1988) Medication outcome in ECT resistant depression. Convulsive Therapy (in press).

\section{Cerebral Ventricular Enlargement in Chronic Schizophrenia}

SIR: Farmer et al (Journal, March 1987, 150, 324330) reported that mean ventricular brain ratio in Type I schizophrenia with positive symptoms was significantly larger at the $P=0.05$ level than that in Type 2 schizophrenia with negative symptoms, in a sample of 35 patients with chronic schizophrenia. This finding is in contrast to the finding of Johnstone et al (1978) and does not support Crow's (1980) proposal that Type 2 schizophrenia is associated with cerebral ventricular enlargement.

In a study conducted at the Psychiatric Unit of the All India Institute of Medical Sciences, New Delhi, 30 patients suffering from schizophrenia with continuous illness of at least two years duration were studied regarding cerebral ventricular enlargement. The diagnosis of schizophrenia was made using Feighner $e t a$ l's research criteria for schizophrenia, and computerised tomography of the brain was performed using CT-1010. Negative and positive symptoms were rated in each patient using the Comprehensive Psychopathological Rating Scale for Schizophrenia (Jacobsson et al, 1978). Of 30 patients, 18 were assessed to have negative symptoms, whereas 12 had positive symptoms. The lateral ventricular size was measured by using free-arm planimetry, and was expressed as ventricular brain ratio. The width of third ventricle and sylvian fissures were measured by using callipers, and were expressed to the nearest millimeter. We found that there was no significant difference between the group of patients with negative symptoms as compared with the group with positive symptoms with regard to ventricular brain ratio $(t=$ $0.14)$, third ventricle $(t=1.05)$, and sylvian fissure $(t=1.27)$.

We are not able to offer any explanation as to why there is such a difference in the findings of different centres regarding association of cerebral ventricular enlargement with Type 2 schizophrenia. We think that Dr Crow's contention regarding the association of ventricular enlargement with Type 2 schizophrenia is not supported by these research findings.

Department of Psychological Medicine

S. K. JAYASWAL

University of Malaya

59100 Kuala Lumpur

Malaysia

H. M. Chawla

R. K. Goulatia

G. S. RAO

All India Institute of Medical Sciences

New Delhi 